\title{
Locality of symmetries generated by nonhereditary, inhomogeneous, and time-dependent recursion operators: a new application for formal symmetries
}

\author{
A. Sergyeyev \\ Silesian University in Opava, Mathematical Institute, \\ Bezručovo nám. 13, 74601 Opava, Czech Republic \\ E-mail: Artur.Sergyeyev@math.slu.cz
}

\begin{abstract}
Using the methods of the theory of formal symmetries, we obtain new easily verifiable sufficient conditions for a recursion operator to produce a hierarchy of local generalized symmetries. An important advantage of our approach is that under certain mild assumptions it allows to bypass the cumbersome check of hereditariness of the recursion operator in question, what is particularly useful for the study of symmetries of newly discovered integrable systems. What is more, unlike the earlier work, the homogeneity of recursion operators and symmetries under a scaling is not assumed as well. An example of nonhereditary recursion operator generating a hierarchy of local symmetries is presented.
\end{abstract}

\section{Introduction}

Since the seminal paper of Olver [1] it became clear that the existence of a recursion operator is one of the most characteristic features of integrable systems of PDEs, cf. e.g. [2, 3, 4] and references therein. Although the integrable hierarchies in $(1+1)$ dimensions usually prove to be local, this is not the case for their recursion operators [2] 8 . Thus, it is natural to ask under which conditions the repeated application of a recursion operator to a local seed symmetry will yield local symmetries corresponding to the higher equations of the hierarchy.

To be more specific, consider first the simplest case of one dependent variable $u$ and a recursion operator of the form

$$
\mathfrak{R}=\sum_{i=0}^{r} a_{i} D^{i}+\sum_{\alpha=1}^{p} G_{\alpha} D^{-1} \circ \gamma_{\alpha},
$$

where $r \geq 0, a_{i}, G_{\alpha}, \gamma_{\alpha}$ belong to the algebra $\mathcal{A}$ of locally analytic functions of $x, t, u, u_{1}, u_{2}, \ldots$ (informally, $\left.u_{j}=\partial^{j} u / \partial x^{j}\right)$, and $D$ is the operator of total $x$-derivative, see below for details.

It is immediate that because of the presence of $D^{-1}$ the application of $\Re$ to a $Q \in \mathcal{A}$ may yield a nonlocal expression, so the question is when the repeated application of $\mathfrak{R}$ will not take us out of $\mathcal{A}$. Clearly, proving that $\mathfrak{R}^{j}(Q)$ are local amounts to proving that $\mathfrak{R}^{j}(Q) \gamma_{\alpha} \in \operatorname{Im} D$ for all $j$ and $\alpha$, where $\operatorname{Im} D$ stands for the image of $D$ in $\mathcal{A}$.

Adler [6] proved this for the recursion operators arising from zero-curvature representations of certain kind, and so did Olver [5] (cf. also Dorfman [4]) for a class of recursion operators, associated with biHamiltonian systems.

Moreover, in the bi-Hamiltonian case there is a very simple and powerful proof of locality of $\mathfrak{R}^{j}(Q)$, kindly communicated to the author by V.V. Sokolov and contained in his unpublished work.

The idea of this proof is as follows: suppose that a recursion operator $\mathfrak{R}$ of the form (1) with $\partial \mathfrak{R} / \partial t=0$ can be written as a ratio of two compatible time-independent Hamiltonian operators: $\mathfrak{R}=\mathfrak{P}_{1} \mathfrak{P}_{2}^{-1}$. Further assume that $\gamma_{\alpha}=\sum_{i=1}^{\infty} c_{\alpha, i} \delta H_{i} / \delta u$ for some constants $c_{\alpha, i}$. Here $\delta / \delta u$ stands for the variational derivative, 
and $H_{i}$ are the Hamiltonians recursively constructed via the Lenard scheme, i.e., $\mathfrak{P}_{1} \delta H_{i+1} / \delta u=\mathfrak{P}_{2} \delta H_{i} / \delta u$, cf. e.g. [2, 4].

Under these assumptions, $H_{i}$ commute with respect to the Poisson brackets associated with $\mathfrak{P}_{1}$ and $\mathfrak{P}_{2}$. Hence if the seed symmetry $Q$ is Hamiltonian with respect to $\mathfrak{P}_{1}$ or $\mathfrak{P}_{2}$, and the respective Hamiltonian is a linear combination of $H_{i}$, then $L_{Q}\left(\delta H_{j} / \delta u\right)=0$ for all $j$, and hence $L_{Q}\left(\gamma_{\alpha}\right)=0$ and $\gamma_{\alpha} Q \in \operatorname{Im} D$, as required. Proceeding inductively, we can prove in this way that all $Q_{j} \equiv \mathfrak{R}^{j}(Q)$ are local. The same argument remains valid for the case of more than one dependent variable.

However, the above results leave open the question of how to prove the locality of symmetries when the recursion operator does not fall into the realm of [6] or we cannot (or, what is typical for the recursion operators of new integrable systems, we do not know how to) write it as a ratio of two Hamiltonian operators. Recently, Sanders and Wang [7] proved a fairly general statement on locality of (1+1)-dimensional hierarchies of evolution systems generated by hereditary recursion operators. They prove that $\mathfrak{R}^{j}(Q) \gamma_{\alpha} \in \operatorname{Im} D$ using the scaling-based arguments and the hereditariness of $\mathfrak{R}$. However, their results are limited to the case when both the recursion operators and symmetries they act on are homogeneous under certain scaling.

In the present paper we suggest a new approach which allows to overcome this limitation and, unlike the earlier work, with minor changes can be applied [9] for the proof of locality of hierarchies generated by master symmetries rather than recursion operators. Moreover, under certain mild assumptions our approach is applicable to nonhereditary recursion operators as well, and, in particular, does not require the check whether the operator in question is hereditary.

We assume that $\gamma_{\alpha}$ are linear combinations of variational derivatives of the so-called canonical conserved densities [10] constructed from $\mathfrak{R}$ (or, more broadly, from a nondegenerate formal symmetry of infinite rank, see Theorem [ below). This is the case for the majority of known today recursion operators, including e.g. those of $\mathrm{KdV}$, mKdV, nonlinear Schrödinger equation and many other integrable evolution equations and systems. For instance, the recursion operator of $\mathrm{KdV}$ equation

$$
\mathfrak{R}=D^{2}+(2 / 3) u D+(1 / 3) u_{1} D^{-1}
$$

obviously is of the form (11) with $p=1$ and $\gamma_{1}=1$, the first nontrivial canonical density is $\rho_{1}=\operatorname{res} \mathfrak{R}^{1 / 2}=u / 3$ and $\gamma_{1}=1=(1 / 3) \delta \rho_{1} / \delta u$. In fact, we failed to find an example that does not satisfy our assumption within the class of recursion operators of the form (11).

Let us stress that for a fairly large number of cases the application of our results does not require the assumption that the recursion operator in question is hereditary. Moreover, the verification of the conditions of our theorems is considerably easier than the check of hereditariness, because the latter usually is extremely cumbersome. This makes our results useful for the study of new recursion operators whose hereditariness is not yet proved. We illustrate this in Section 5 by proving the locality of a hierarchy generated by the recursion operator (18).

Below we present our main results in their simplest form, i.e., for the case of a single dependent variable $u$. In this case the canonical densities are $\rho_{i}=\operatorname{res} \mathfrak{R}^{i / r}$ for $i=-1,1,2, \ldots$, and $\rho_{0}=(1 / r) a_{r-1} / a_{r}$.

Theorem 1 Let $\mathfrak{R}$ (11) with $r>0$ be hereditary on the linear span of $\mathfrak{R}^{j}\left(Q_{0}\right), j=0,1,2 \ldots$, for a $Q_{0} \in \mathcal{A}$ and satisfy $L_{Q_{0}}(\Re)=0$, and let there exist the functions $c_{\alpha, i}(t)$ such that

$$
\gamma_{\alpha}=\sum_{i=-1}^{\infty} c_{\alpha, i}(t) \delta \rho_{i} / \delta u
$$

for all $\alpha=1, \ldots, p$, where $\rho_{i}$ are canonical densities associated with $\Re$.

Then $Q_{j}=\mathfrak{R}^{j}\left(Q_{0}\right)$ are local, i.e., $Q_{j} \in \mathcal{A}$, for all $j \in \mathbb{N}$.

We also can use the scaling-based arguments in order to simultaneously prove the hereditariness of $\mathfrak{R}$ and the locality of symmetries. 
Theorem 2 Consider an operator $\mathfrak{R}$ of the form (1) with $r>0$, and let there exist the functions $c_{\alpha, i}(t)$ such that (2) holds for all $\alpha=1, \ldots, p$. Let there also exist $Q_{0} \in \mathcal{A}$ and $S \in \mathcal{A}$ such that ord $Q_{0} \geq 2$, $\partial Q_{0} / \partial t=0, \partial S / \partial t=0, L_{Q_{0}}(\mathfrak{R})=0,\left[S, Q_{0}\right]=\nu Q_{0}, \nu=$ const, $\nu \neq 0$, and $L_{S}(\Re)=\zeta \Re, \zeta=$ const, $\zeta \neq 0$.

Then $Q_{j}=\mathfrak{R}^{j}\left(Q_{0}\right)$ are local, $L_{Q_{j}}(\mathfrak{R})=0,\left[Q_{i}, Q_{j}\right]=0$ for all $i, j=0,1,2 \ldots$, and $\mathfrak{R}$ is hereditary on the linear span of $Q_{j}, j=0,1,2, \ldots$

Moreover, under certain conditions we can drop the requirement of hereditariness of $\mathfrak{R}$, but then we have to fix an evolution equation

$$
u_{t}=F\left(x, t, u, \ldots, u_{n}\right), n \geq 2 .
$$

Let $n_{0}=\left\{\begin{array}{l}1-j, \text { where } j \text { is the greatest integer such that } \\ \partial F / \partial u_{i} \text { for } i=n-j, \ldots, n \text { depend on } x \text { and } t \text { only, } \\ 2 \text { otherwise. }\end{array}\right.$

Theorem 3 Let (3) with $n \geq 2$ have a symmetry $Q_{0} \in \mathcal{A}$ such that ord $Q_{0} \geq \max \left(n_{0}, 0\right)$ and $\partial Q_{0} / \partial t=0$, and a recursion operator $\mathfrak{R}$ of the form (1) with $r>0$ such that $\partial \mathfrak{R} / \partial t=0$. Further assume that (2) holds and $c_{\alpha, i}(t) \equiv 0$ for all $i>n-n_{0}-1$ and all $\alpha=1, \ldots, p$.

Then $Q_{j}=\mathfrak{R}^{j}\left(Q_{0}\right)$ are local for all $j \in \mathbb{N}$.

Note that if $\partial F / \partial t=0$, we can take $F$ for $Q_{0}$ in Theorem 3

Let us also mention that if $\partial F / \partial t=0$, then the densities $\rho_{i}$ for $i=-1, \ldots, n-n_{0}-1$, unlike their counterparts with $i>n-n_{0}-1$, can be computed directly from $F$ [10, 12]: $\rho_{0}=(1 / n) \partial F / \partial u_{n-1}\left(\partial F / \partial u_{n}\right)^{-1}$, and for $j=-1,1 \ldots, n-n_{0}-1 \rho_{j}=$ res $\mathfrak{R}^{j / r}$ is a linear combination of $\tilde{\rho}_{k}, k=-1,1, \ldots, j$, where $\tilde{\rho}_{k}=\operatorname{res}\left(F^{\prime}\right)^{k / n}, F^{\prime}=\sum_{i=0}^{n} \partial F / \partial u_{i} D^{i}$.

In the rest of the paper we present the extension of Theorems 1- 3 to the case of more than one dependent variable, see Theorems 4 6] below, along with the complete proofs and examples, see Section 5 for the latter. In this case the coefficients of recursion operators are matrices, so the nondegeneracy and diagonalizability issues come into play. Nevertheless, the extra conditions that appear in our theorems in the case of more than one dependent variable are easy to verify, and they are satisfied for a large number of interesting examples.

Our technique can be applied not only to the recursion operators but also to the so-called inverse Noether operators [14] that map symmetries to cosymmetries. Namely, our Corollaries 2 and 3 enable one to prove the existence of infinite number of local cosymmetries, and eventually of local conserved densities, generated using these operators. Note that whenever exist, the symplectic operators of integrable evolution systems automatically are [14] inverse Noether operators for these systems.

\section{Preliminaries}

Consider an algebra $\mathcal{A}_{j}$ of locally analytic functions of $x, t, \boldsymbol{u}, \boldsymbol{u}_{1}, \ldots, \boldsymbol{u}_{j}$, where $\boldsymbol{u}_{k}=\left(u_{k}^{1}, \ldots, u_{k}^{s}\right)^{T}$ are vectors, $\boldsymbol{u}_{0} \equiv \boldsymbol{u}$, and let $\mathcal{A}=\cup_{j=0}^{\infty} \mathcal{A}_{j}$. We shall call the elements of $\mathcal{A}$ local functions, cf. e.g. [2, 11]. Let us further introduce [2, 11] a derivation $D \equiv D_{x}=\partial / \partial x+\sum_{j=0}^{\infty} \boldsymbol{u}_{j+1} \partial / \partial \boldsymbol{u}_{j}$, the variational derivative $\delta / \delta \boldsymbol{u}=\sum_{j=0}^{\infty}(-D)^{j} \partial / \partial \boldsymbol{u}_{j}$ and the operator of directional derivative of $\vec{f} \in \mathcal{A}^{q}: \vec{f}^{\prime}=\sum_{i=0}^{\infty} \partial \vec{f} / \partial \boldsymbol{u}_{i} D^{i}$. In what follows we shall see that $x$ will play the role of space variable, $D$ will be the total $x$-derivative, and, if we specify an evolution system $\partial \boldsymbol{u} / \partial t=\boldsymbol{F}\left(x, t, \boldsymbol{u}, \ldots, \boldsymbol{u}_{n}\right)$, then $t$ becomes an evolution parameter.

The Lie bracket, see e.g. [2], given for any $\boldsymbol{P}, \boldsymbol{Q} \in \mathcal{A}^{s}$ by $[\boldsymbol{P}, \boldsymbol{Q}]=\boldsymbol{P}^{\prime}[\boldsymbol{Q}]-\boldsymbol{Q}^{\prime}[\boldsymbol{P}]$ makes the linear space $\mathcal{A}^{s}$ of $s$-component vectors whose components belong to $\mathcal{A}$ into a Lie algebra, and enables us to define the Lie derivative of $\boldsymbol{R} \in \mathcal{A}^{s}$ along $\boldsymbol{Q} \in \mathcal{A}^{s}$ by setting $L_{\boldsymbol{Q}}(\boldsymbol{R})=[\boldsymbol{Q}, \boldsymbol{R}]$.

Now consider [2, 10, 12] the set $\operatorname{Mat}_{q}(\mathcal{A}) \llbracket D^{-1} \rrbracket$ of formal series of the form $\mathfrak{H}=\sum_{j=-\infty}^{k} h_{j} D^{j}$, where $h_{j}$ are $q \times q$ matrices with entries from $\mathcal{A}$. We shall write $\mathcal{A} \llbracket D^{-1} \rrbracket$ instead of $\operatorname{Mat}_{1}(\mathcal{A}) \llbracket D^{-1} \rrbracket$ for short. The greatest $m$ such that $h_{m} \neq 0$ is called the degree of formal series $\mathfrak{H}$ and is denoted as deg $\mathfrak{H}$, cf. e.g. 2, 10, 12, Clearly, $\boldsymbol{G}^{\prime} \in \operatorname{Mat}_{s}(\mathcal{A}) \llbracket D^{-1} \rrbracket$ for any $\boldsymbol{G} \in \mathcal{A}^{s}$, so we shall define the order of $\boldsymbol{G} \in \mathcal{A}^{s}$ as ord $\boldsymbol{G}=\operatorname{deg} \boldsymbol{G}^{\prime}$.

A formal series $\mathfrak{H}=\sum_{j=-\infty}^{m} h_{j} D^{j} \in \operatorname{Mat}_{q}(\mathcal{A}) \llbracket D^{-1} \rrbracket$ of degree $m$ is called nondegenerate [10 if det $h_{m} \neq 0$. For $\mathfrak{H}=\sum_{j=-\infty}^{m} h_{j} D^{j} \in \mathcal{A} \llbracket D^{-1} \rrbracket, \operatorname{deg} \mathfrak{H}=m$, define its residue as res $\mathfrak{H}=h_{-1}$, and its logarithmic 
residue as res $\ln \mathfrak{H}=h_{m-1} / h_{m}$, see e.g. $\llbracket 10$. For $\mathfrak{H}=\sum_{j=-\infty}^{m} h_{j} D^{j} \in \operatorname{Mat}_{q}(\mathcal{A}) \llbracket D^{-1} \rrbracket$ and $\boldsymbol{Q} \in \mathcal{A}^{s}$ set $\mathfrak{H}^{\prime}[\boldsymbol{Q}]=\sum_{j=-\infty}^{m} h_{j}^{\prime}[\boldsymbol{Q}] D^{j}$.

The multiplication law, which is nothing but the generalized Leibniz rule, cf. e.g. [2],

$$
a D^{i} \circ b D^{j}=a \sum_{q=0}^{\infty} \frac{i(i-1) \cdots(i-q+1)}{q !} D^{q}(b) D^{i+j-q},
$$

extended by linearity to the whole $\operatorname{Mat}_{q}(\mathcal{A}) \llbracket D^{-1} \rrbracket$, makes this set into an algebra, and the commutator $[\mathfrak{A}, \mathfrak{B}]=\mathfrak{A} \circ \mathfrak{B}-\mathfrak{B} \circ \mathfrak{A}$ further makes it into a Lie algebra. Below we omit $\circ$ unless this leads to confusion.

For any nondegenerate $\mathfrak{H} \in \operatorname{Mat}_{q}(\mathcal{A}) \llbracket D^{-1} \rrbracket$ we can $\llbracket 10$ define its inverse $\mathfrak{H}^{-1} \in \operatorname{Mat}_{q}(\mathcal{A}) \llbracket D^{-1} \rrbracket$ such that $\mathfrak{H} \circ \mathfrak{H}^{-1}=\mathfrak{H}^{-1} \circ \mathfrak{H}=\mathbb{I}$, where $\mathbb{I}$ is a $q \times q$ unit matrix. Moreover [2, 10, 11, 12, for any $\mathfrak{H} \in \mathcal{A} \llbracket D^{-1} \rrbracket$, $\operatorname{deg} \mathfrak{H}=m \neq 0$, we can define its $m$ th root $\mathfrak{H}^{1 / m}$ and its fractional powers $\mathfrak{H}^{j / m}=\left(\mathfrak{H}^{1 / m}\right)^{j}$, and these fractional powers commute: $\left[\mathfrak{H}^{i / m}, \mathfrak{H}^{j / m}\right]=0$ for all $i, j \in \mathbb{Z}[2$, 10.

Define 22 the Lie derivative of $\mathfrak{H} \in \operatorname{Mat}_{s}(\mathcal{A}) \llbracket D^{-1} \rrbracket$ along $\boldsymbol{Q} \in \mathcal{A}^{s}$ as $L_{\boldsymbol{Q}}(\mathfrak{H})=\mathfrak{H}^{\prime}[\boldsymbol{Q}]-\left[\boldsymbol{Q}^{\prime}, \mathfrak{H}\right]$. A formal series $\mathfrak{H} \in \operatorname{Mat}_{s}(\mathcal{A}) \llbracket D^{-1} \rrbracket$ is called [2, 10, 11, 12] a formal symmetry of rank $m$ (respectively of infinite rank) for an evolution system $\partial \boldsymbol{u} / \partial \tau=\boldsymbol{Q}, \boldsymbol{Q} \in \mathcal{A}^{s}$, where $\tau$ is an evolution parameter other than $t$, if $\operatorname{deg} L_{\boldsymbol{Q}}(\mathfrak{H}) \leq \operatorname{deg} \mathfrak{H}+\operatorname{deg} \boldsymbol{Q}^{\prime}-m$ (resp. $\left.L_{\boldsymbol{Q}}(\mathfrak{H})=0\right)$. The formal symmetry for an evolution system $\partial \boldsymbol{u} / \partial t=\boldsymbol{Q}$ is defined in a similar way: we only should [10, 11, 12] replace $L_{\boldsymbol{Q}}(\mathfrak{H})$ by $\partial \mathfrak{H} / \partial t+L_{\boldsymbol{Q}}(\mathfrak{H})$ in the above definition.

Lemma 1 Consider $\mathfrak{H}=\sum_{j=-\infty}^{m} h_{j} D^{j} \in \operatorname{Mat}_{s}(\mathcal{A}) \llbracket D^{-1} \rrbracket$ such that $\operatorname{deg} \mathfrak{H}=m \neq 0$ and the matrix $h_{m}$ has exactly $s$ distinct eigenvalues $\lambda_{i}$. Let $\Gamma$ be a matrix bringing $h_{m}$ into the diagonal form: $h_{m}=\Gamma^{-1} \Lambda \Gamma$, $\Lambda=\operatorname{diag}\left(\lambda_{1}, \ldots, \lambda_{s}\right)$.

Then there exists a unique formal series $\mathfrak{T}=\Gamma+\sum_{j=-1}^{-\infty} \Gamma_{j} \Gamma D^{j}$ with the property $\operatorname{diag} \Gamma_{j}=0, j=$ $-1,-2, \ldots$, such that all coefficients of the formal series $\tilde{\mathfrak{H}}=\mathfrak{T H T}^{-1}$ are diagonal matrices; $\Gamma_{j}$ are $s \times s$ matrices with entries from $\mathcal{A}$.

Moreover, for any $\boldsymbol{Q} \in \mathcal{A}^{\text {s }}$, ord $\boldsymbol{Q} \geq 1$, such that $\mathfrak{H}$ is the formal symmetry of infinite rank for the equation $\partial \boldsymbol{u} / \partial \tau=\boldsymbol{Q}$ (resp. $\partial \boldsymbol{u} / \partial t=\boldsymbol{Q}$ ), all coefficients of the formal series $\mathfrak{V} \equiv \mathfrak{T} \boldsymbol{Q}^{\prime} \mathfrak{T}^{-1}+\mathfrak{T}^{\prime}[\boldsymbol{Q}] \mathfrak{T}^{-1}$ (resp. $\left.\mathfrak{W} \equiv \mathfrak{T} \boldsymbol{Q}^{\prime} \mathfrak{T}^{-1}+\left(\partial \mathfrak{T} / \partial t+\mathfrak{T}^{\prime}[\boldsymbol{Q}]\right) \mathfrak{T}^{-1}\right)$ are diagonal matrices.

Proof. Equating the coefficients at the powers of $D$ in $\mathfrak{T} \mathfrak{H}=\tilde{\mathfrak{H}} \mathfrak{T}$ enables us to find $\Gamma_{j}$ and the coefficients of $\tilde{\mathfrak{H}}$, whence we readily infer the existence and uniqueness of $\mathfrak{T}=\Gamma+\sum_{j=-1}^{-\infty} \Gamma_{j} \Gamma D^{j}$ such that $\operatorname{diag} \Gamma_{j}=0$ and all coefficients of $\tilde{\mathfrak{H}}=\mathfrak{T} \mathfrak{H} \mathfrak{T}^{-1}$ are diagonal matrices, cf. the proof of Proposition 2.1 in [10]. We use here the well-known fact that for any diagonal $s \times s$ matrix $\Lambda$ with $s$ distinct eigenvalues and for any $s \times s$ matrix $\Omega$ with zero diagonal entries (we write this as $\operatorname{diag} \Omega=0$ ) the equation $[\Lambda, \Psi]=\Omega$ has a unique solution in the class of $s \times s$ matrices $\Psi$ with $\operatorname{diag} \Psi=0$, cf. e.g. [10].

Further observe that the transformation $\mathfrak{H} \rightarrow \tilde{\mathfrak{H}}$ and $Q^{\prime} \rightarrow \mathfrak{V}$ (resp. $Q^{\prime} \rightarrow \mathfrak{W}$ for the equation $\left.\boldsymbol{u}_{t}=\boldsymbol{Q}\right)$ takes the equation $L_{\boldsymbol{Q}}(\mathfrak{H})=0\left(\right.$ resp. $\left.\partial \mathfrak{H} / \partial t+L_{\boldsymbol{Q}}(\mathfrak{H})=0\right)$ to $\tilde{\mathfrak{H}}^{\prime}[\boldsymbol{Q}]-[\mathfrak{V}, \tilde{\mathfrak{H}}]=0$ (resp. to $\left.\partial \tilde{\mathfrak{H}} / \partial t+\tilde{\mathfrak{H}}^{\prime}[\boldsymbol{Q}]-[\mathfrak{W}, \tilde{\mathfrak{H}}]=0\right)$. Using the above-mentioned result on solutions of $[\Lambda, \Psi]=\Omega$ and successively solving the equations for the coefficients of $\mathfrak{V}$ (resp. $\mathfrak{W}$ ) that arise from equating to zero the coefficients at powers of $D$ on the left-hand side of the equation $\tilde{\mathfrak{H}}^{\prime}[\boldsymbol{Q}]-[\mathfrak{V}, \tilde{\mathfrak{H}}]=0\left(\operatorname{resp} . \partial \tilde{\mathfrak{H}} / \partial t+\tilde{\mathfrak{H}}^{\prime}[\boldsymbol{Q}]-[\mathfrak{W}, \tilde{\mathfrak{H}}]=0\right)$, we readily see that all coefficients of $\mathfrak{V}$ (resp. $\mathfrak{W}$ ) are diagonal matrices. $\square$ Following [10], consider the canonical densities associated with $\mathfrak{H}: \rho_{j}^{a}=\operatorname{res}\left(\left(\mathfrak{T} \mathfrak{H T}^{-1}\right)_{a a}\right)^{j /|m|}, j=-1,1,2, \ldots, a=1, \ldots, s$, and $\rho_{0}^{a}=\operatorname{res} \ln \left(\left(\mathfrak{T} \mathfrak{H T}^{-1}\right)_{a a}\right)^{1 /|m|}, a=1, \ldots, s$, where the subscript ' $a a$ ' means taking the $(a, a)$-th entry of the matrix. Lemma 1 and the results of [10] readily imply the following assertion.

Corollary 1 Under the assumptions of Lemma 1, suppose that $\mathfrak{H}$ is nondegenerate. Then the quantities $\rho_{j}^{a}, a=1, \ldots, s, j=-1,0,1,2, \ldots$ are conserved densities for the equation $\boldsymbol{u}_{\tau}=\boldsymbol{Q}\left(\right.$ resp. $\left.\boldsymbol{u}_{t}=\boldsymbol{Q}\right)$, i.e., $\rho_{j}^{\prime a}[\boldsymbol{Q}] \in \operatorname{Im} D\left(\right.$ resp. $\left.\partial \rho_{j}^{a} / \partial t+\rho_{j}^{\prime a}[\boldsymbol{Q}] \in \operatorname{Im} D\right)$.

Thus, the canonical densities associated with $\mathfrak{H}$ are common conserved densities for all systems of the form $\boldsymbol{u}_{\tau}=\boldsymbol{Q}$ (or $\boldsymbol{u}_{t}=\boldsymbol{Q}$ ) admitting $\mathfrak{H}$ as a formal symmetry of infinite rank. Moreover, the formal series $\mathfrak{T}$ that diagonalizes $\mathfrak{H}$ simultaneously diagonalizes the formal series $\mathfrak{V}$ (or $\mathfrak{W}$ ) for all these systems. 


\section{The main result}

Consider an operator

$$
\mathfrak{R}=\sum_{i=0}^{r} a_{i} D^{i}+\sum_{\alpha=1}^{p} \boldsymbol{G}_{\alpha} \otimes D^{-1} \circ \gamma_{\alpha},
$$

where $r \geq 0, a_{i}$ are $s \times s$ matrices with entries from $\mathcal{A}$, and $\boldsymbol{G}_{\alpha}, \boldsymbol{\gamma}_{\alpha} \in \mathcal{A}^{s}$, i.e., $\boldsymbol{G}_{\alpha}, \boldsymbol{\gamma}_{\alpha}$ are $s$-component vectors with entries from $\mathcal{A}$.

The Lie derivative $L_{\boldsymbol{Q}}(\mathfrak{R})$ of $\mathfrak{R}$ along $\boldsymbol{Q} \in \mathcal{A}^{s}$ is given [2] by $L_{\boldsymbol{Q}}(\mathfrak{R})=\mathfrak{R}^{\prime}[\boldsymbol{Q}]-\left[\boldsymbol{Q}^{\prime}, \mathfrak{R}\right]$, that is, by the very same formulae as for the elements of $\operatorname{Mat}_{s}(\mathcal{A}) \llbracket D^{-1} \rrbracket$, because using the generalized Leibniz rule (4) we always can rewrite $\mathfrak{R}$ as infinite formal series in powers of $D$, and thus consider it as an element of $\operatorname{Mat}_{s}(\mathcal{A}) \llbracket D^{-1} \rrbracket$.

An operator $\mathfrak{R}$ is called, see e.g. [2], a recursion operator for an evolution system $\boldsymbol{u}_{t}=\boldsymbol{F}\left(x, t, \boldsymbol{u}, \ldots, \boldsymbol{u}_{n}\right)$ (respectively $\boldsymbol{u}_{\tau}=\boldsymbol{F}\left(x, t, \boldsymbol{u}, \ldots, \boldsymbol{u}_{n}\right)$, where $\tau$ is an evolution parameter other than $t$ ), if it is a formal symmetry of infinite rank for this system, i.e. if it satisfies the equation $\partial \mathfrak{R} / \partial t+L_{\boldsymbol{F}}(\mathfrak{R})=0\left(\right.$ resp. $\left.L_{\boldsymbol{F}}(\mathfrak{R})=0\right)$.

Note that nearly all known today recursion operators of integrable $(1+1)$-dimensional evolution systems are [7, 8] of the form (5), with only a few exceptions, see e.g. 16, 17, 18,

Recall that a linear operator $\mathfrak{R}$ is said [14] to be hereditary (or Nijenhuis [4]) on a linear space $\mathcal{L}$, if for all $Q \in \mathcal{L}$

$$
L_{\mathfrak{R}(\boldsymbol{Q})}(\mathfrak{R})=\mathfrak{R} \circ L_{\boldsymbol{Q}}(\mathfrak{R}) .
$$

In what follows by saying that a recursion operator is hereditary without specifying $\mathcal{L}$ we shall mean that it is hereditary on its whole domain of definition, cf. [7. If $\mathfrak{R}$ is hereditary on $\mathcal{L}$, then [14 for any $\boldsymbol{Q} \in \mathcal{L}$ we have $\left[\Re^{i}(\boldsymbol{Q}), \mathfrak{R}^{j}(\boldsymbol{Q})\right]=0, i, j=0,1,2, \ldots$

Let $\mathcal{S}(\mathfrak{R}, \boldsymbol{Q})$ denote the linear span of $\mathfrak{R}^{i}(\boldsymbol{Q}), i=0,1,2, \ldots$ It is immediate from (6) that $L_{\mathfrak{R}^{i}(\boldsymbol{Q})}(\mathfrak{R})=0$ for all $i=0,1,2, \ldots$ if and only if $L_{\boldsymbol{Q}}(\mathfrak{R})=0$ and $\mathfrak{R}$ is hereditary on $\mathcal{S}(\mathfrak{R}, \boldsymbol{Q})$. In view of this, we have $\left[\Re^{i}(\boldsymbol{Q}), \mathfrak{R}^{j}(\boldsymbol{Q})\right]=0$ for all $i, j=0,1,2, \ldots$, provided

$$
L_{\mathfrak{R}^{i}(\boldsymbol{Q})}(\mathfrak{R})=0 \text { for all } i=0,1,2, \ldots
$$

In view of this, the results of Sanders and Wang [7] on locality of hierarchies of symmetries generated by recursion operators obviously remain valid if the requirement of hereditariness of recursion operator on its whole domain of definition is replaced by (7) with $i=1,2, \ldots$. What is more, (7) can be often proved using simple scaling arguments (which are, however, quite different from those of [7]), see Theorem 5 below.

Let us now turn to a general situation when (7) holds, but, unlike [7, no assumption on scaling properties of $\mathfrak{R}$ and $\boldsymbol{Q}$ is made.

Theorem 4 Let $\mathfrak{R}$ (5) with $r>0$ be hereditary on $\mathcal{S}\left(\mathfrak{R}, \boldsymbol{Q}_{0}\right)$ for a $\boldsymbol{Q}_{0} \in \mathcal{A}^{s}$. Suppose that $L_{\boldsymbol{Q}_{0}}(\mathfrak{R})=0$, $\operatorname{det} a_{r} \neq 0$, the matrix $a_{r}$ has $s$ distinct eigenvalues, and there exist the functions $c_{\alpha, i, a}(t)$ such that

$$
\gamma_{\alpha}=\sum_{i=-1}^{\infty} \sum_{a=1}^{s} c_{\alpha, i, a}(t) \delta \rho_{i}^{a} / \delta \boldsymbol{u}
$$

for all $\alpha=1, \ldots, p$, where $\rho_{i}^{a}$ are canonical densities associated with $\mathfrak{R}$.

Then $\boldsymbol{Q}_{j}=\mathfrak{R}^{j}\left(\boldsymbol{Q}_{0}\right)$ are local, that is, $\boldsymbol{Q}_{j} \in \mathcal{A}^{s}$, for all $j \in \mathbb{N}$, and commute: $\left[\boldsymbol{Q}_{i}, \boldsymbol{Q}_{j}\right]=0$ for all $i, j=0,1,2 \ldots$

Proof. As $\mathfrak{R}$ is hereditary on $\mathcal{S}\left(\mathfrak{R}, \boldsymbol{Q}_{0}\right)$, we have $L_{\mathfrak{R}^{j}\left(\boldsymbol{Q}_{0}\right)}(\mathfrak{R})=0$, and thus $\mathfrak{R}$ is a formal symmetry of infinite rank for all evolution systems of the form $\partial \boldsymbol{u} / \partial t_{j}=\boldsymbol{Q}_{j}, j=0,1,2, \ldots$. Hence, if $r>0$, then the canonical densities $\rho_{i}^{a}$ for $i=-1,0,1,2, \ldots$ and $a=1, \ldots, s$ are conserved densities for all these systems. If $\boldsymbol{Q}_{j}$ is local, this means that $\rho_{i}^{\prime a}\left[\boldsymbol{Q}_{j}\right] \in \operatorname{Im} D$. But $\rho_{i}^{\prime a}\left[\boldsymbol{Q}_{j}\right] \in \operatorname{Im} D$ if and only if $\delta \rho_{i}^{a} / \delta \boldsymbol{u} \cdot \boldsymbol{Q}_{j} \in \operatorname{Im} D$, where - stands for the scalar product of $s$-component vectors.

Hence, $\boldsymbol{\gamma}_{\alpha} \cdot \boldsymbol{Q}_{j} \in \operatorname{Im} D$ by (8), because $\delta \rho_{i}^{a} / \delta \boldsymbol{u} \cdot \boldsymbol{Q}_{j} \in \operatorname{Im} D$ by the above. Thus, $D^{-1}\left(\boldsymbol{\gamma}_{\alpha} \cdot \boldsymbol{Q}_{j}\right) \in \mathcal{A}$ for all $\alpha=1, \ldots, p$, and $\boldsymbol{Q}_{j+1}$ is local, if so is $\boldsymbol{Q}_{j}$. The induction on $j$ starting from $j=0$ completes the proof. 
Remark 1. The condition $\operatorname{det} a_{r} \neq 0$ can be relaxed. Indeed, if $\operatorname{det} a_{r}=0$, then the matrix $a_{r}$ has precisely one zero eigenvalue, say $\lambda_{b}: \lambda_{b}=0$. Then we should just require that $m \equiv \operatorname{deg}\left(\mathfrak{T R T}^{-1}\right)_{b b} \neq 0$ and replace the densities $\rho_{i}^{b}$ by the following ones: $\tilde{\rho}_{j}^{b}=\operatorname{res}\left(\left(\mathfrak{T R T} \mathfrak{T}^{-1}\right)_{b b}\right)^{j /|m|}$ for $j=-1,1,2, \ldots$, and $\tilde{\rho}_{0}^{b}=\operatorname{res} \ln \left(\left(\mathfrak{T R T}^{-1}\right)_{b b}\right)^{1 /|m|}$.

Remark 2. If we have $\gamma_{\alpha}=\sum_{i=-1}^{\infty} c_{\alpha, i}(t) \delta \tilde{\rho}_{i} / \delta \boldsymbol{u}$ for all $\alpha=1, \ldots, p$, where $\tilde{\rho}_{i}=\operatorname{tr}$ res $\mathfrak{R}^{i}, i=-1,1,2, \ldots$, $\tilde{\rho}_{0}=\operatorname{tr}$ res $\ln \mathfrak{R}$, then the assumption of Theorem 1 that $a_{r}$ has $s$ distinct eigenvalues can be dropped. Furthermore, if $c_{\alpha,-1}(t)=c_{\alpha, 0}(t)=0$ for all $\alpha=1, \ldots, p$, then we can also drop the assumption det $a_{r} \neq 0$.

As we have already mentioned above, it is possible to prove that $\mathfrak{R}$ is hereditary on $\mathcal{S}\left(\mathfrak{R}, \boldsymbol{Q}_{0}\right)$ using the scaling-based arguments. The locality of $\boldsymbol{Q}_{i}$ then follows by the same argument as in the proof of Theorem 4 ,

Theorem 5 Let $\mathfrak{R}$ of the form (5) with $r>0$ be such that $\partial \mathfrak{R} / \partial t=0$, $\operatorname{det} a_{r} \neq 0$, the matrix $a_{r}$ has $s$ distinct eigenvalues, and let there exist the functions $c_{\alpha, i, a}(t)$ such that (8) holds for all $\alpha=1, \ldots, p$. Let there also exist $\boldsymbol{Q}_{0} \in \mathcal{A}^{s}$ and $\boldsymbol{S} \in \mathcal{A}^{s}$ such that $q_{0} \equiv$ ord $\boldsymbol{Q}_{0} \geq 2, \partial \boldsymbol{Q}_{0} / \partial t=0$, the matrix $\partial \boldsymbol{Q}_{0} / \partial \boldsymbol{u}_{q_{0}}$ is nondegenerate and has $s$ distinct eigenvalues, $\partial \boldsymbol{S} / \partial t=0, L_{\boldsymbol{Q}_{0}}(\mathfrak{R})=0,\left[\boldsymbol{S}, \boldsymbol{Q}_{0}\right]=\nu \boldsymbol{Q}_{0}$, where $\nu$ is a nonzero constant. Further assume that $L_{\boldsymbol{S}}\left(\mathfrak{T}^{-1} c \tilde{\mathfrak{R}}^{1 / r} \mathfrak{T}\right)=\mu \mathfrak{T}^{-1} c \mathfrak{\Re}^{1 / r} \mathfrak{T}$ for any matrix $c=\operatorname{diag}\left(c_{1}, \ldots, c_{s}\right)$, $c_{i}=$ const, where $\mu$ is a nonzero constant independent of $c_{1}, \ldots, c_{s}, \tilde{\mathfrak{R}}=\mathfrak{T} \mathfrak{R T} \mathfrak{T}^{-1}$, and $\mathfrak{T}$ is the formal series constructed in Lemma 1 for $\mathfrak{H}=\mathfrak{R}$.

Then $\boldsymbol{Q}_{j}=\mathfrak{R}^{j}\left(\boldsymbol{Q}_{0}\right)$ are local, satisfy $L_{\boldsymbol{Q}_{j}}(\mathfrak{R})=0$, and commute, that is, $\left[\boldsymbol{Q}_{i}, \boldsymbol{Q}_{j}\right]=0$, for all $i, j=0,1,2 \ldots$, and $\mathfrak{R}$ is hereditary on the linear span of $\boldsymbol{Q}_{j}, j=0,1,2, \ldots$

Proof. The condition $L_{\boldsymbol{Q}_{0}}(\Re)=0$ implies (cf. [10, 12]) that

$$
\boldsymbol{Q}_{0}^{\prime}=\sum_{j=2}^{q_{0}} \mathfrak{T}^{-1} d_{j}^{\left(\boldsymbol{Q}_{0}\right)} \tilde{\mathfrak{R}}^{j / r} \mathfrak{T}+\mathfrak{B}_{\boldsymbol{Q}_{0}},
$$

where $\mathfrak{B}_{\boldsymbol{Q}_{0}} \in \operatorname{Mat}_{s}(\mathcal{A}) \llbracket D^{-1} \rrbracket$ is a formal series with diagonal leading coefficient, and $\operatorname{deg} \mathfrak{B}_{\boldsymbol{Q}_{0}}<2 ; d_{j}^{\left(\boldsymbol{Q}_{0}\right)}$ are constant diagonal $s \times s$ matrices.

From (92) it is immediate that $\nu=\mu q_{0}$. It is also clear that $L_{\boldsymbol{S}}(\mathfrak{R})=\zeta \mathfrak{R}$, where $\zeta=r \mu$. Using this formula and $\left[\boldsymbol{S}, \boldsymbol{Q}_{0}\right]=\nu \boldsymbol{Q}_{0}=\mu q_{0} \boldsymbol{Q}_{0}$ yields $\left[\boldsymbol{S}, \boldsymbol{Q}_{j}\right]=(\nu+j \eta) \boldsymbol{Q}_{j}=\mu\left(q_{0}+r j\right) \boldsymbol{Q}_{j}$, whence $L_{\boldsymbol{S}}\left(L_{\boldsymbol{Q}_{j}}(\mathfrak{R})\right)=$ $L_{\boldsymbol{Q}_{j}}\left(L_{\boldsymbol{S}}(\mathfrak{R})\right)+\mu\left(q_{0}+r j\right) L_{\boldsymbol{Q}_{j}}(\mathfrak{R})=\mu\left(r(j+1)+q_{0}\right) L_{\boldsymbol{Q}_{j}}(\mathfrak{R})$.

Using the Leibniz rule for the Lie derivative and $L_{\boldsymbol{Q}_{0}}(\mathfrak{R})=0$, we obtain $\left[\boldsymbol{Q}_{0}, \boldsymbol{Q}_{j}\right]=L_{\boldsymbol{Q}_{0}}\left(\boldsymbol{Q}_{j}\right)=$ $L_{\boldsymbol{Q}_{0}}\left(\mathfrak{R}^{j} \boldsymbol{Q}_{0}\right)=L_{\boldsymbol{Q}_{0}}\left(\mathfrak{R}^{j}\right) \boldsymbol{Q}_{0}+\mathfrak{R}^{j}\left[\boldsymbol{Q}_{0}, \boldsymbol{Q}_{0}\right]=L_{\boldsymbol{Q}_{0}}\left(\mathfrak{R}^{j}\right) \boldsymbol{Q}_{0}=0$, whence $L_{\boldsymbol{Q}_{0}}\left(L_{\boldsymbol{Q}_{j}}(\mathfrak{R})\right)=L_{\boldsymbol{Q}_{j}}\left(L_{\boldsymbol{Q}_{0}}(\mathfrak{R})\right)=0$.

Therefore, $L_{\boldsymbol{Q}_{j}}(\mathfrak{R})$ is a formal symmetry of infinite rank for the system $\boldsymbol{u}_{\tau}=\boldsymbol{Q}_{0}$. Now we can proceed inductively. Assume that we have already proved that $\boldsymbol{Q}_{j}$ is local (for $j=1$ this readily follows from our assumptions). Then the coefficients of the formal series $L_{\boldsymbol{Q}_{j}}(\mathfrak{R})$ are local too, and hence $L_{\boldsymbol{Q}_{j}}(\mathfrak{R})=$ $\sum_{l=-\infty}^{k} \mathfrak{T}^{-1} h_{l}^{\left(\boldsymbol{Q}_{j}\right)} \tilde{\mathfrak{R}}^{l / r} \mathfrak{T}$, where $h_{l}^{\left(\boldsymbol{Q}_{j}\right)}$ are constant diagonal $s \times s$ matrices and $k<r(j+1)+q_{0}$, cf. [10, 11].

Using this formula and our assumptions on $\boldsymbol{S}, \boldsymbol{Q}_{0}$, and $\mathfrak{R}$, we readily find that $L_{\boldsymbol{S}}\left(L_{\boldsymbol{Q}_{j}}(\mathfrak{R})\right)=\mu k L_{\boldsymbol{Q}_{0}}(\mathfrak{R})$. But, as $k<r(j+1)+q_{0}$, this contradicts our earlier result $L_{\boldsymbol{S}}\left(L_{\boldsymbol{Q}_{0}}(\mathfrak{R})\right)=\mu\left(q_{0}+(j+1) r\right) L_{\boldsymbol{Q}_{j}}(\mathfrak{R})$, unless $L_{\boldsymbol{Q}_{j}}(\mathfrak{R})=0$. Hence, $L_{\boldsymbol{Q}_{j}}(\mathfrak{R})=0$. Then, by the same argument as in the proof of Theorem $4, \boldsymbol{Q}_{j+1}$ is local, and we can repeat the above reasoning with $j$ replaced by $j+1$.

Thus, we have $L_{\boldsymbol{Q}_{j}}(\mathfrak{R})=0$ for all $j \in \mathbb{N}$, and therefore $\mathfrak{R}$ is hereditary on the linear span of $\boldsymbol{Q}_{j}=\mathfrak{R}^{j}\left(\boldsymbol{Q}_{0}\right)$, $j=0,1,2 \ldots$, so $\boldsymbol{Q}_{j}$ commute for all $j=0,1,2, \ldots$.

Note that the conditions of Theorem 5 are easy to verify in spite of their complicated appearance. In particular, in the most common situation when $\boldsymbol{S}=x \boldsymbol{u}_{1}+\Lambda \boldsymbol{u}$, where $\Lambda$ is a constant diagonal matrix, we usually can choose the coefficients of $\mathfrak{T}$ so that $L_{\boldsymbol{S}}(\mathfrak{T})=0$, and then in order to verify the equality

$$
L_{\boldsymbol{S}}\left(\mathfrak{T}^{-1} c \tilde{\mathfrak{R}}^{1 / r} \mathfrak{T}\right)=\mu \mathfrak{T}^{-1} c \tilde{\mathfrak{R}}^{1 / r} \mathfrak{T}
$$

for any constant diagonal matrix $c$ it suffices to check that $L_{\boldsymbol{S}}(\mathfrak{R})=\zeta \mathfrak{R}$ for some nonzero constant $\zeta$. Moreover, for $s=1$ we have $\mathfrak{T}=1$, and it suffices to check that $L_{\boldsymbol{S}}(\mathfrak{R})=\zeta \mathfrak{R}$ for some $\zeta=$ const $\neq 0$ in order to prove (10) even if $\boldsymbol{S}$ is not of the form $x \boldsymbol{u}_{1}+\Lambda \boldsymbol{u}$. 
Consider now an application of our results to operators other than recursion operators. Recall that $\mathfrak{K} \in \operatorname{Mat}_{s}(\mathcal{A}) \llbracket D^{-1} \rrbracket$ is called [14] an inverse Noether operator for an evolution system $\boldsymbol{u}_{t}=\boldsymbol{F}\left(x, t, \boldsymbol{u}, \ldots, \boldsymbol{u}_{n}\right)$ (respectively $\boldsymbol{u}_{\tau}=\boldsymbol{F}\left(x, t, \boldsymbol{u}, \ldots, \boldsymbol{u}_{n}\right)$, where $\tau$ is an evolution parameter other than $t$ ), if it satisfies the equation $\partial \mathfrak{K} / \partial t+\mathfrak{K}^{\prime}[\boldsymbol{F}]+\left(\boldsymbol{F}^{\prime}\right)^{\dagger} \circ \mathfrak{K}+\mathfrak{K} \circ \boldsymbol{F}^{\prime}=0\left(\right.$ resp. $\left.\mathfrak{K}^{\prime}[\boldsymbol{F}]+\left(\boldsymbol{F}^{\prime}\right)^{\dagger} \circ \mathfrak{K}+\mathfrak{K} \circ \boldsymbol{F}^{\prime}=0\right)$. We shall restrict ourselves to considering the inverse Noether operators of the form

$$
\mathfrak{K}=\sum_{i=0}^{h} b_{i} D^{i}+\sum_{\alpha=1}^{l} \boldsymbol{\eta}_{\alpha} \otimes D^{-1} \circ \boldsymbol{\zeta}_{\alpha}
$$

where $b_{i}$ are $s \times s$ matrices with entries from $\mathcal{A}$, and $\boldsymbol{\eta}_{\alpha}, \boldsymbol{\zeta}_{\alpha} \in \mathcal{A}^{s}$. The majority of known today inverse Noether operators of integrable (1+1)-dimensional evolution systems indeed are of the form (11), see e.g. [8].

Our Theorems 4 and 5 readily imply the following assertion.

Corollary 2 Let a system $\boldsymbol{u}_{t}=\boldsymbol{F}\left(x, t, \boldsymbol{u}, \ldots, \boldsymbol{u}_{n}\right)$ or $\boldsymbol{u}_{\tau}=\boldsymbol{F}\left(x, t, \boldsymbol{u}, \ldots, \boldsymbol{u}_{n}\right), \tau \neq t$, have a recursion operator $\mathfrak{R}$ meeting the requirements of Theorem 4 or 5 and an inverse Noether operator $\mathfrak{K}$ of the form (11) with $h \geq 0$. Assume that there exist the functions $d_{\alpha, i, a}(t)$ such that $\boldsymbol{\zeta}_{\alpha}=\sum_{i=-1}^{\infty} \sum_{a=1}^{s} d_{\alpha, i, a}(t) \delta \rho_{i}^{a} / \delta \boldsymbol{u}$ for all $\alpha=1, \ldots, l$, where $\rho_{i}^{a}$ are canonical densities associated with $\mathfrak{R}$.

Then $\boldsymbol{\chi}_{j} \equiv \mathfrak{K}\left(\boldsymbol{Q}_{j}\right)$ are local (i.e., $\left.\boldsymbol{\chi}_{j} \in \mathcal{A}^{s}\right)$ for all $j=0,1,2, \ldots$

Proof. We have $\boldsymbol{\zeta}_{\alpha} \cdot \boldsymbol{Q}_{j} \in \operatorname{Im} D$ by (8), because $\delta \rho_{i}^{a} / \delta \boldsymbol{u} \cdot \boldsymbol{Q}_{j} \in \operatorname{Im} D$, cf. the proof of Theorem 4 . Therefore, $D^{-1}\left(\boldsymbol{\zeta}_{\alpha} \cdot \boldsymbol{Q}_{j}\right) \in \mathcal{A}$ for all $\alpha=1, \ldots, p$ and $j=0,1,2 \ldots$, and thus $\mathfrak{K}\left(\boldsymbol{Q}_{j}\right) \in \mathcal{A}^{s}$.

\section{Locality of hierarchies of symmetries generated by nonhereditary recursion operators}

Even if a recursion operator is not hereditary, it still can generate a hierarchy of local symmetries. A simple example is given (see [15] for a more complicated example with $s=3$ ) by

$$
\mathfrak{R}=\left(\begin{array}{cc}
D & u_{1} D^{-1} \\
0 & -D+v
\end{array}\right)
$$

For $\boldsymbol{K}=(u, 0)^{T}$ we have $L_{\boldsymbol{K}}(\mathfrak{R})=0$ but $L_{\mathfrak{R}(\boldsymbol{K})}(\mathfrak{R}) \neq 0$, so $\mathfrak{R}$ (12) is not hereditary. Nevertheless, all $\boldsymbol{K}_{i}=\mathfrak{R}^{i}(\boldsymbol{K})$ obviously are local: assuming that $D^{-1}(0)=0$, see e.g. [16, 19, 20] for more details on defining $D^{-1}$, we readily find that $\boldsymbol{K}_{i}=\left(u_{i}, 0\right)^{T}$.

While in the above example the locality of symmetries was obvious, this is not the case in general. Fortunately, there is a way around: in Theorem [6] below we present the sufficient conditions ensuring the locality of $\boldsymbol{Q}_{i}=\mathfrak{R}^{i}\left(\boldsymbol{Q}_{0}\right)$ without assuming that $\mathfrak{R}$ is hereditary. To this end we need, however, to fix an evolution system

$$
\partial \boldsymbol{u} / \partial t=\boldsymbol{F}\left(x, t, \boldsymbol{u}, \boldsymbol{u}_{1}, \ldots, \boldsymbol{u}_{n}\right), \quad n \geq 2,
$$

with $\partial \boldsymbol{F} / \partial \boldsymbol{u}_{n} \neq 0$.

If the matrix $\partial \boldsymbol{F} / \partial \boldsymbol{u}_{n}$ is nondegenerate and has $s$ distinct eigenvalues, we shall say that (13) is a nondegenerate weakly diagonalizable (NWD) system. The properties of such systems were studied e.g. in 10, 12, 21. Clearly, for $s=1$ any system (13) with $\partial \boldsymbol{F} / \partial \boldsymbol{u}_{n} \neq 0$ is NWD.

Recall that $\boldsymbol{G} \in \mathcal{A}^{s}$ is called [2, 12] a symmetry for (13) if $\partial \boldsymbol{G} / \partial t+[\boldsymbol{F}, \boldsymbol{G}]=0$, where $[\cdot, \cdot]$ is the Lie bracket, defined above.

Let $n_{0}=\left\{\begin{array}{l}1-j, \text { where } j \text { is the greatest number such that } \\ \partial \boldsymbol{F} / \partial \boldsymbol{u}_{i} \text { for } i=n-j, \ldots, n \text { depend on } x \text { and } t \text { only, } \\ 2 \text { otherwise. }\end{array}\right.$

Now assume that (13) is NWD, $\mathfrak{R}$ is the recursion operator for (13), and $\boldsymbol{Q}_{0} \in \mathcal{A}^{s}$ is a symmetry for (13), and hence all $\boldsymbol{Q}_{i}=\mathfrak{R}^{i}\left(\boldsymbol{Q}_{0}\right)$ are symmetries for (13). Further assume that $\partial \boldsymbol{Q}_{0} / \partial t=0$ and that $\mathfrak{R}$ is such that this implies $\partial \boldsymbol{Q}_{j} / \partial t=0$ for all $j \in \mathbb{N}$. 
Further assume that (13) has a time-independent $(\partial \mathfrak{L} / \partial t=0)$ nondegenerate formal symmetry $\mathfrak{L}=$ $\sum_{j=-\infty}^{m} g_{i} D^{i}$ of infinite rank and of degree $m>0$, and the matrix $g_{m}$ has $s$ distinct eigenvalues $\lambda_{i}$. Clearly, if $r>0, \partial \mathfrak{R} / \partial t=0$, det $a_{r} \neq 0$ and $a_{r}$ has $s$ distinct eigenvalues, we can take $\mathfrak{R}$ for $\mathfrak{L}$.

Then, using the diagonalizing transformation $\mathfrak{T}$ for $\mathfrak{H}=\mathfrak{L}$ from Lemma 1 we readily see that if $\boldsymbol{Q}_{j}$ is local, then $\boldsymbol{Q}_{j}^{\prime}$ is a formal symmetry of degree $q_{j} \equiv$ ord $\boldsymbol{Q}_{j}$ and of rank $q_{j}-n_{0}+2$ for (13), cf. e.g. 10, 21]. Hence we

have $\boldsymbol{Q}_{j}^{\prime}=\sum_{k=n_{0}}^{q_{j}} \mathfrak{T}^{-1} d_{k}^{\left(\boldsymbol{Q}_{j}\right)} \tilde{\mathfrak{L}}^{j / m} \mathfrak{T}+\mathfrak{B}_{\boldsymbol{Q}_{j}}$, where $\operatorname{deg} \mathfrak{B}_{\boldsymbol{Q}_{j}}<n_{0}, \tilde{\mathfrak{L}}=\mathfrak{T} \mathfrak{L} \mathfrak{T}^{-1}$ and $d_{k}^{\left(\boldsymbol{Q}_{j}\right)}$ are constant diagonal $s \times s$ matrices, cf. e.g. 10, 12, 21]. Using this result, we can show that $\operatorname{deg} L_{\boldsymbol{Q}_{j}}(\mathfrak{L}) \leq m-1+2-n-n_{0}$, hence $\mathfrak{L}$ is a formal symmetry of rank $q_{j}+n-n_{0}+1$ for the system $\boldsymbol{u}_{t_{j}}=\boldsymbol{Q}_{j}$, where $q_{j}=$ ord $\boldsymbol{Q}_{j}, j=0,1,2 \ldots$ Indeed, for $s=1$ this holds by Lemma 11 of Sokolov [11, and for $s>1$ this is proved along the same lines as in [1].

Hence, by the results of [10], for $a=1, \ldots, s$ and $b=-1,0,1, \ldots, n-n_{0}-1$ the canonical densities $\rho_{b}^{a}$ associated with $\mathfrak{L}$ are conserved densities for the systems $\partial \boldsymbol{u} / \partial t_{j}=\boldsymbol{Q}_{j}, j=0,1,2, \ldots$, i.e., $\rho_{b}^{\prime a}\left[\boldsymbol{Q}_{j}\right] \in \operatorname{Im} D$, and the locality of all $\boldsymbol{Q}_{k}, k \in \mathbb{N}$, is proved by induction on $k$ as in the proof of Theorem 4 so we arrive at the following result.

Theorem 6 Let an NWD system (13) with $n \geq 2$ have a time-independent nondegenerate formal symmetry $\mathfrak{L}$ of degree $m>0$ and of infinite rank, and let the leading coefficient of $\mathfrak{L}$ have $s$ distinct eigenvalues. Further assume that (13) has a time-independent symmetry $\boldsymbol{Q}_{0} \in \mathcal{A}^{s}$, ord $\boldsymbol{Q}_{0} \geq \max \left(n_{0}, 0\right)$, and a recursion operator $\mathfrak{R}$ of the form (5) with $r \geq 0$ such that $\boldsymbol{Q}_{i}=\mathfrak{R}^{i}\left(\boldsymbol{Q}_{0}\right)$ are time-independent for all $i \in \mathbb{N}$. Suppose that there exist the functions $c_{\alpha, i, a}(t)$ such that

$$
\gamma_{\alpha}=\sum_{i=-1}^{n-n_{0}-1} \sum_{a=1}^{s} c_{\alpha, i, a}(t) \delta \rho_{i}^{a} / \delta \boldsymbol{u}
$$

for all $\alpha=1, \ldots, p$, where $\rho_{i}^{a}$ are canonical densities associated with $\mathfrak{L}$.

Then $\boldsymbol{Q}_{j}$ are local, that $i s, \boldsymbol{Q}_{j} \in \mathcal{A}^{s}$, for all $j \in \mathbb{N}$.

Let us mention that, exactly as in the case of one dependent variable $u$, if $\partial \boldsymbol{F} / \partial t=0$, then the densities $\rho_{i}^{a}$ for $i=-1, \ldots, n-n_{0}-1$ can be computed directly from $\boldsymbol{F}$. Namely, up to multiplication by a constant and adding a linear combination of $\rho_{k}^{a}, k<j$, we have $\rho_{0}^{a}=\operatorname{res} \ln \left(\left(D_{t}(\mathfrak{T}) \mathfrak{T}^{-1}+\mathfrak{T}^{\prime} \mathfrak{T}^{-1}\right)_{a a}\right)^{1 / n}$, and $\rho_{j}^{a}=\operatorname{res}\left(\left(D_{t}(\mathfrak{T}) \mathfrak{T}^{-1}+\mathfrak{T} \boldsymbol{F}^{\prime} \mathfrak{T}^{-1}\right)_{a a}\right)^{j / n}, j=-1,1,2, \ldots, n-n_{0}-1, a=1, \ldots, s$.

In complete analogy with Corollary 2 , we have the following result.

Corollary 3 Under the assumptions of Theorem [6, let (13) have an inverse Noether operator $\mathfrak{K}$ of the form (11) with $h \geq 0$. Assume that there exist the functions $d_{\alpha, i, a}(t)$ such that

$$
\boldsymbol{\zeta}_{\alpha}=\sum_{i=-1}^{n-n_{0}-1} \sum_{a=1}^{s} d_{\alpha, i, a}(t) \delta \rho_{i}^{a} / \delta \boldsymbol{u}
$$

for all $\alpha=1, \ldots, l$, where $\rho_{i}^{a}$ are canonical densities associated with $\mathfrak{L}$.

Then $\boldsymbol{\eta}_{j}=\mathfrak{K}\left(\boldsymbol{Q}_{j}\right)$ are local, i.e., $\boldsymbol{\eta}_{j} \in \mathcal{A}^{s}$, for all $j=0,1,2, \ldots$

\section{Examples}

Let us agree that for scalar $\boldsymbol{u}$, i.e., for $s=1$, we shall write $u, u_{j}, \gamma_{\alpha}, F, Q$ instead of $\boldsymbol{u}, \boldsymbol{u}_{j}, \boldsymbol{\gamma}_{\alpha}, \boldsymbol{F}, \boldsymbol{Q}$, as we already did in Introduction.

Consider first the generalized Korteweg-de Vries equation 22

$$
u_{t}=-u_{3}-6 u u_{1}-6 f(t) u+x\left(\dot{f}(t)+12(f(t))^{2}\right),
$$

where $f(t)$ is an arbitrary smooth function of time $t$. 
This equation has 22] a hereditary recursion operator

$$
\mathfrak{R}=(g(t))^{2}\left(D^{2}+4(u-x f(t))\right)+2 g(t)\left(u_{1}-f(t)\right) D^{-1} \circ g(t),
$$

where $g(t)=\exp \left(6 \int_{t_{0}}^{t} f\left(t^{\prime}\right) d t^{\prime}\right)$. Clearly, $\mathfrak{R}$ is of the form (15) with $p=1$ and $\gamma_{1}=g(t)$. Upon setting $\mathfrak{H}=\mathfrak{R}$ we find that $\rho_{1}=2 g(t)(u-x f(t))$, so $\delta \rho_{1} / \delta u=2 g(t)$. Taking $c_{1,1}(t)=1 / 2$, we see that the requirements of Theorem $\square$ are met, and hence the repeated application of $\mathfrak{R}$ to the seed symmetry $Q=g(t)\left(u_{1}-f(t)\right)$ yields an infinite hierarchy of local symmetries for (14).

Let now $s=2, \boldsymbol{u}=(u, v)^{T}$, and consider the so-called DS IV system 23] (also known as the HirotaSatsuma 24] system)

$$
\begin{aligned}
& u_{t}=u_{3} / 2+3 u u_{1}-6 v v_{1}, \\
& v_{t}=-v_{3}-3 u v_{1} .
\end{aligned}
$$

and its recursion operator, see e.g. [26],

$$
\begin{aligned}
& \mathfrak{R}=\left(\begin{array}{cc}
\mathfrak{B} & -5 v D^{2}-4 v_{1} D-v_{2}-4 u v \\
-(5 / 2) v_{1} D-3 v_{2} & \mathfrak{C}
\end{array}\right) \\
& +\boldsymbol{G}_{1} \otimes D^{-1} \circ \gamma_{1}+\boldsymbol{G}_{2} \otimes D^{-1} \circ \gamma_{2},
\end{aligned}
$$

where $\mathfrak{B}=D^{4} / 2+2 u D^{2}+3 u_{1} D+2 u_{2}+4\left(u^{2}-v^{2}\right), \mathfrak{C}=-D^{4}-4 u D^{2}-2 u_{1} D-4 v^{2}, \boldsymbol{G}_{1}=\left(u_{1}, v_{1}\right)^{T}$, $\gamma_{1}=(u,-2 v), \boldsymbol{G}_{2}=\left(u_{3} / 2+3 u u_{1}-6 v v_{1},-v_{3}-3 u v_{1}\right)^{T}, \gamma_{2}=(1,0)$.

Set $\boldsymbol{Q}_{0}=\left(u_{3} / 2+3 u u_{1}-6 v v_{1},-v_{3}-3 u v_{1}\right)^{T}$ and $\boldsymbol{S}=x \boldsymbol{u}_{1}+2 \boldsymbol{u}$. Then all requirements of Theorem 5 (and of Theorem 4 too, as $\mathfrak{R}$ is hereditary) are met. In particular, there exist the constants $c_{1,1,1}, c_{1,3,1}$, $c_{1,3,2}$ such that $\gamma_{1}=c_{1,1,1} \delta \rho_{1}^{1} / \delta \boldsymbol{u}+c_{1,3,1} \delta \rho_{3}^{1} / \delta \boldsymbol{u}+c_{1,3,2} \delta \rho_{3}^{2} / \delta \boldsymbol{u}$ and $\gamma_{2}=c_{2,1,1} \delta \rho_{1}^{1} / \delta \boldsymbol{u}$. Thus, by Theorem $\mathbf{5}$ all $\boldsymbol{Q}_{j}=\mathfrak{R}^{j}\left(\boldsymbol{Q}_{0}\right)$ are local.

Moreover, the system (15) has an inverse Noether operator which also is symplectic, cf. e.g. 223, 25],

$$
\mathfrak{K}=\left(\begin{array}{cc}
D / 2 & 0 \\
0 & -2 D
\end{array}\right)+\gamma_{1} \otimes D^{-1} \circ \gamma_{2}+\gamma_{2} \otimes D^{-1} \circ \gamma_{1}
$$

It is immediate that the requirements of Corollary 2 are met, and thus the cosymmetries $\boldsymbol{\chi}_{j}=\mathfrak{K}\left(\boldsymbol{Q}_{j}\right)$ are local for all $j=0,1,2, \ldots$.

Consider now the Calogero-Degasperis-Fokas equation [27, 28]

$$
u_{t}=u_{3}-u_{1}^{3} / 8+(a \exp (u)+b \exp (-u)+c) u_{1}
$$

where $a, b, c$ are arbitrary constants. It has the recursion operator [28]

$$
\begin{aligned}
& \Re=D^{2}-\frac{u_{1}^{2}}{4}+\frac{2}{3}(a \exp (u)+b \exp (-u)+c) \\
& +\frac{u_{1}}{3} D^{-1} \circ\left(\frac{3 u_{2}}{4}+a \exp (u)-b \exp (-u)\right) .
\end{aligned}
$$

For $\mathfrak{H}=\mathfrak{R}$ we have $\rho_{1}^{1}=-u_{1}^{2} / 8+(1 / 3)(a \exp (u)+b \exp (-u)+c)$, so $\gamma_{1}=3 \delta \rho_{1}^{1} / \delta u$. Thus, $\mathfrak{R}$ meets the requirements of Theorem 6 (and of Theorem 4 as well, because $\mathfrak{R}$ is hereditary), and hence all $Q_{j}=\mathfrak{R}^{j}\left(u_{1}\right)$, $j \in \mathbb{N}$, are local.

Let again $s=2, \boldsymbol{u}=(u, v)^{T}$. Consider the operator [29]

$$
\mathfrak{R}=\left(\begin{array}{cc}
D^{2} / 4+v-u^{2} / 4 & 3 D / 2+u / 2 \\
(3 / 4) v_{1} & D^{2}+u D+v
\end{array}\right)+\sum_{\alpha=1}^{2} \boldsymbol{G}_{\alpha} \otimes D^{-1} \circ \gamma_{\alpha},
$$

where $\boldsymbol{G}_{1}=\left(2 v_{1}, v_{2}+u v_{1}\right)^{T}, \boldsymbol{\gamma}_{1}=(1 / 2,0), \boldsymbol{G}_{1}=\left(u_{1}, v_{1}\right)^{T}, \boldsymbol{\gamma}_{2}=(-u / 4,1 / 2)$. This is a recursion operator for the NWD system

$$
\begin{aligned}
& u_{t}=u_{3} / 4+(3 / 2)\left(v_{2}+v u_{1}+u v_{1}\right)-(3 / 8) u^{2} u_{1} \\
& v_{t}=v_{3}+(3 / 2)\left(u v_{2}+v v_{1}\right)+(3 / 8) u^{2} v_{1}
\end{aligned}
$$


We have $\rho_{0}^{2}=u$ and $\rho_{1}^{1}=v / 2-u^{2} / 8$, so $\gamma_{1}=(1 / 2) \delta \rho_{0}^{1} / \delta \boldsymbol{u}$ and $\gamma_{2}=\delta \rho_{1}^{1} / \delta \boldsymbol{u}$. Hence, $\mathfrak{R}$ meets the requirements of Theorem $\left[\right.$ for $\boldsymbol{F}=\boldsymbol{Q}_{0}=\mathfrak{R}\left(\boldsymbol{u}_{1}\right)$, where $\boldsymbol{F}$ stands for the right-hand side of (19), and thus all $\boldsymbol{Q}_{j}=\mathfrak{R}^{j+1}\left(\boldsymbol{u}_{1}\right), j \in \mathbb{N}$, are local.

Let us stress that (14), (17) and (19) have no scaling symmetry of the form used in [7, so in these cases it is impossible prove the locality of hierarchies of symmetries using the results of [7].

\section{Acknowledgements}

I am sincerely grateful to Profs. B. Fuchssteiner and V.V. Sokolov for stimulating discussions. I am also pleased to thank Dr. M. Marvan for kindly reading the manuscript of the present paper and making a number of useful remarks. I acknowledge with gratitude the kind hospitality of organizers of Banach Center Workshop "Multi-Hamiltonian Structures: Algebraic and Geometric Aspects" in Bȩdlewo, Poland, where some of the results of this paper were presented.

This research was supported by DFG via Graduiertenkolleg "Geometrie und Nichtlineare Analysis" of Institute für Mathematik of Humboldt-Universität zu Berlin, Germany, where the author held a postdoctoral fellowship. I also acknowledge the partial support from the Ministry of Education, Youth and Sports of Czech Republic under Grant MSM:J10/98:192400002, and from the Czech Grant Agency under Grant No. 201/00/0724.

\section{References}

[1] Olver, P. J.: Evolution equations possessing infinitely many symmetries, J. Math. Phys. 18 (1977) $1212-1215$.

[2] Olver, P.J.: Applications of Lie Groups to Differential Equations, Springer, New York, 1993.

[3] Błaszak, M.: Multi-Hamiltonian Theory of Dynamical Systems, Springer, Heidelberg, 1998.

[4] Dorfman, I.: Dirac Structures and Integrability of Nonlinear Evolution Equations, John Wiley \& Sons, Chichester, 1993.

[5] Olver, P. J.: Bi-Hamiltonian systems, in: Ordinary and partial differential equations (Dundee, 1986), Longman, Harlow, 1987, pp. 176-193.

[6] Adler, V.E.: Lie-algebraic approach to nonlocal symmetries of integrable systems, Theor. Math. Phys. 89 (1992), 1239-1248.

[7] Sanders, J.A. and Wang, J.P.: Integrable systems and their recursion operators, Nonlinear Analysis 47 (2001) 5213-5240.

[8] Wang, J.P.: A list of $1+1$ dimensional integrable equations and their properties. J. Nonlinear Math. Phys. 9 (2002), suppl. 1, 213-233.

[9] Sergyeyev, A.: On sufficient conditions of locality for hierarchies of symmetries of evolution systems, Rep. Math. Phys. 50 (2002), 307-314.

[10] Mikhailov, A.V., Shabat, A.B., and Yamilov, R.I.: The symmetry approach to classification of nonlinear equations. Complete lists of integrable systems, Russ. Math. Surv. 42(1987), no.4, 1-63.

[11] Sokolov, V.V.: Symmetries of evolution equations, Russ. Math. Surv. 43(1988), no. 5, 165-204.

[12] Mikhailov, A.V., Shabat, A.B., and Sokolov, V.V.: The symmetry approach to classification of integrable equations, in: V.E. Zakharov (ed), What is Integrability?, Springer, New York, 1991, pp. 115-184.

[13] Mikhailov, A.V., Yamilov, R.I.: Towards classification of (2+1)-dimensional integrable equations. Integrability conditions. I, J. Phys. A: Math. Gen. 31 (1998) 6707-6715. 
[14] Fuchssteiner, B. and Fokas, A.S.: Symplectic structures, their Bäcklund transformations and hereditary symmetries, Physica D 4 (1981/82), 47-66.

[15] Li, Y.S., Zeng, Y.B.: On the nonhereditary recursion operator and the constraint on the potential associated with the Giachetti-Johnson equation and its gauge equivalent Yang equation, J. Phys. A: Math. Gen. 23 (1990) 721-733.

[16] Guthrie, G.A.: Recursion operators and non-local symmetries, Proc. Roy. Soc. London Ser. A 446 (1994), no. 1926, 107-114.

[17] Marvan, M.: Another look on recursion operator, in: J. Janyška, I. Kolář and J. Slovák (eds), Differential Geometry and Applications (Brno, 1995), Masaryk University, Brno, 1996, pp. 393-402, available online at http://www.emis.de/proceedings

[18] Karasu (Kalkanli), A., Karasu, A., and Sakovich, S. Yu.: A strange recursion operator for a new integrable system of coupled Korteweg-de Vries equations, Preprint nlin.SI/0203036, 2002.

[19] Sanders, J.A. and Wang, J.P.: On recursion operators, Physica D 149 (2001), 1-10.

[20] Sergyeyev, A.: On recursion operators and nonlocal symmetries of evolution equations, in: D. Krupka (ed), Proc. Sem. Diff. Geom., Silesian University in Opava, 2000, pp. 159-173 (also available as an e-print nlin.SI/0012011)

[21] Sergyeyev, A.: On homogeneous symmetries for evolution systems with constraints, Rendiconti del Circolo Matematico di Palermo Serie II Suppl. 69 (2002), 219-231.

[22] Chou, T.: Symmetries and a hierarchy of the general KdV equation, J. Phys. A: Math. Gen. 20 (1987), 359-366.

[23] Drinfeld, V.G., Sokolov, V.V.: Lie algebras and equations of Korteweg-de Vries type, J. Sov. Math. 30 (1985), 1975-2036.

[24] Satsuma, J. and Hirota, R.: A coupled KdV equation is one case of the four-reduction of the KP hierarchy, J. Phys. Soc. Japan 51 (1982), no. 10, 3390-3397.

[25] Fuchssteiner, B.: The Lie algebra structure of degenerate Hamiltonian and bi-Hamiltonian systems, Progr. Theor. Phys. 68 (1982), 1082-1104.

[26] Gürses, M., Karasu, A. and Sokolov, V.V.: On construction of recursion operators from Lax representation, J. Math. Phys. 40 (1999), 6473-6490.

[27] Calogero, F. and Degasperis, A.: Reduction technique for matrix nonlinear evolution equations solvable by the spectral transform, J. Math. Phys. 22 (1981), 23-31.

[28] Fokas, A.S.: A symmetry approach to exactly solvable evolution equations, J. Math. Phys. 21 (1980) $1318-1325$.

[29] Błaszak, M.: On the construction of recursion operator and algebra of symmetries for field and lattice systems, Rep. Math. Phys. 48 (2000), 27-38. 